\title{
Hypergraph Model of Multi-residue Interactions in Proteins: Sequentially-Constrained Partitioning Algorithms for Optimization of Site-Directed Protein Recombination
}

\author{
Xiaoduan Ye ${ }^{1}$, Alan M. Friedman ${ }^{2}$, and Chris Bailey-Kellogg ${ }^{1}$ \\ 1 Department of Computer Science, Dartmouth College, \\ 6211 Sudikoff Laboratory, Hanover NH 03755, USA \\ \{ye, cbk\}@cs.dartmouth.edu \\ 2 Department of Biological Sciences and Purdue Cancer Center, \\ Purdue University, West Lafayette, IN 47907, USA \\ afried@purdue.edu
}

\begin{abstract}
Relationships among amino acids determine stability and function and are also constrained by evolutionary history. We develop a probabilistic hypergraph model of residue relationships that generalizes traditional pairwise contact potentials to account for the statistics of multi-residue interactions. Using this model, we detected non-random associations in protein families and in the protein database. We also use this model in optimizing site-directed recombination experiments to preserve significant interactions and thereby increase the frequency of generating useful recombinants. We formulate the optimization as a sequentially-constrained hypergraph partitioning problem; the quality of recombinant libraries wrt a set of breakpoints is characterized by the total perturbation to edge weights. We prove this problem to be NP-hard in general, but develop exact and heuristic polynomial-time algorithms for a number of important cases. Application to the beta-lactamase family demonstrates the utility of our algorithms in planning site-directed recombination.
\end{abstract}

\section{Introduction}

The non-random association of amino acids, as expressed in pairwise potentials, has been usefully applied in a number of situations. Such pairwise contact potentials [1,2 play a large role in evaluating quality of models in protein structure prediction [3,4,5, 6. It has been suggested, however, that "it is unlikely that purely pairwise potentials are sufficient for structure prediction" [7, 8 .

To better model evolutionary relationships that determine protein stability and functionality, it may be necessary to capture the higher-order interactions that are ignored in simple pairwise models (Fig. 1(a)). Researchers have begun to demonstrate the importance of accounting for higher-order terms. A statistical pseudo-potential based on four-body nearest neighbor interactions (as 


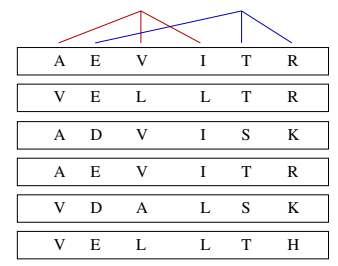

(a)

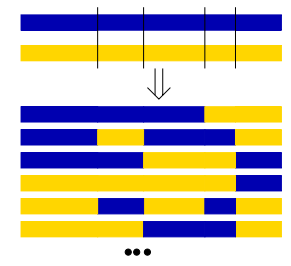

(b)

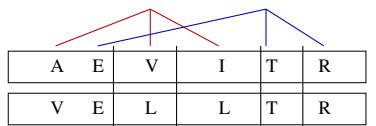

(c)

Fig. 1. Hypergraph model of evolutionary interactions, and effects of site-directed protein recombination. (a) Higher-order evolutionary interactions (here, order-3) determining protein stability and function are observed in the statistics of "hyperconservation" of mutually interacting positions. The left edge is dominated by Ala,Val,Ile and Val,Leu,Leu interactions, while the right is dominated by Glu,Thr,Arg and Asp,Ser,Lys ones. The interactions are modeled as edges in a hypergraph with weights evaluating the degree of hyperconservation of an interaction, both generally in the protein database and specific to a particular family. (b) Site-directed recombination mixes and matches sequential fragments of homologous parents to construct a library of hybrids with the same basic structure but somewhat different sequences and thus different functions. (c) Site-directed recombination perturbs edges that cross one or more breakpoints. The difference in edge weights derived for the parents and those derived for the hybrids indicates the effect of the perturbation on maintenance of evolutionarily favorable interactions.

determined by Delaunay tessellations) has successfully predicted changes in free energy caused by hydrophobic core mutations [8]. Similar formulations have been used to discriminate native from non-native protein conformations [9]. Geometrically less restricted higher-order interactions have also been utilized for recognition of native-like protein structures [10. Recent work on correlated mutation analysis has moved from identifying pairwise correlations [11 to determining clusters or cliques of mutually-dependent residues that identify subclasses within a protein family and provide mechanistic insights into function [12, 13].

This paper develops a rigorous basis for representing multi-order interactions within a protein family. We generalize the traditional representations of sequence information in terms of single-position conservation and structural interactions in terms of pairwise contacts. Instead, we define a hypergraph model in which edges represent pairwise and higher-order residue interactions, while edge weights represent the degree of "hyperconservation" of the interacting residues (Sec. 2). Hyperconservation can reveal significant residue interactions both within members of the family (arising from structural and functional constraints) and generally common to all proteins (arising from general properties of the amino acids). We then combine family-specific and database-wide statistics with suitable weighting (Sec. 2.1), ensure non-redundancy of the information in super- and sub-edges with a multi-order potential score (Sec. 2.2$)$, and derive edge weights by mean potential scores (Sec. 2.3). Application of our approach to beta-lactamases (Sec. 4) shows that the effect of non-redundant higher-order terms is significant and can be effectively handled by our model. 
Protein recombination in vitro (Fig. 1(b)) enables the design of protein variants with favorable properties and novel enzymatic activities, as well as the exploration of protein sequence-structure-function relationships (see e.g. [14, 15, 16, 17, 18, 19, 20, 21, 22 ). In this approach, libraries of hybrid proteins are generated either by stochastic enzymatic reactions or intentional selection of breakpoints. Hybrids with unusual properties can either be identified by largescale genetic screening and selection, or many hybrids can be evaluated individually to determine detailed sequence-function relationships for understanding and/or rational engineering. We focus here on site-directed recombination, in which parent genes are recombined at specified breakpoint locations, yielding hybrids in which different sequence fragments (between the breakpoints) can come from different parents. Both screening/selection and investigational experiments benefit from recombination that preserves the most essential structural and functional features while still allowing variation. In order to enhance the success of this approach, it is necessary to choose breakpoint locations that optimize preservation of these features.

The labs of Mayo and Arnold [18,23 have established criteria for nondisruption of contacting residue pairs and demonstrated the relationship between non-disruption and functional hybrids [18]. There is an on-going search for algorithms to select breakpoints for recombination based on non-disruption [23, 24], although none has yet been experimentally validated. Optimizing multi-order interactions after recombination (Fig. 1(c)) should help identify the best recombinants and thus the best locations for breakpoints. In support of this optimization, we develop criteria to evaluate the quality of hybrid libraries by considering the effects of recombination on edge weights (Sec. 2.4). We then formulate the optimal selection of breakpoint locations as a sequentially-constrained hypergraph partitioning problem (Sec. 3), prove it to be NP-hard in general (Sec. 3.1), develop exact and heuristic algorithms for a number of important cases (Secs. 3.2 3.5), and demonstrate their practical effectiveness in design of recombination experiments for members of the beta-lactamase family (Sec. 41).

\section{A Hypergraph Model of Evolutionary Interactions}

In order to more completely model statistical interactions in a protein, it is necessary to move beyond single-position sequence conservation and pairwise structural contact. We model a protein and its reference structure with a weighted hypergraph $G=(V, E, w)$, where vertices $V=\left\{v_{1}, v_{2}, \cdots, v_{|V|}\right\}$ represent residue positions in sequential order on the backbone, edges $E \subseteq 2^{V}$ represent mutually interacting sets of vertices, and weight function $w: E \rightarrow \mathbb{R}$ represents the relative significance of edges. We construct an order- $c$ edge $e=\left\langle v_{1}, v_{2}, \cdots, v_{c}\right\rangle$ for each set of residues (listed in sequential order for convenience) that are in mutual contact; this construction can readily be extended to capture other forms of interaction, e.g. long-range interaction of non-contacting residues due to electrostatics. Note that subsets of vertices associated with a higher-order edge form lower-order edges. When we need to specify the exact order $c$ of edges in a hypergraph, we use 
notation $G_{c}=\left(V, E_{c}, w\right)$. Since lower-order edges can be regarded as a special kind of higher-order ones, $G_{c}$ includes "virtual" lower-order edges.

The definition of the edge weight is key to effective use of the hypergraph model. In the case where the protein is a member of a family with presumed similar structures, edge weights can be evaluated both from the general database and specific to the family. There are many observed residue values (across the family or database) for the vertices of any given edge. We thus build up to an edge weight by first estimating the probability of the residue values, then decomposing the probability to ensure non-redundant information among multi-order edges for the same positions. Finally we determine the effect on the pattern of these values due to recombination according to a set of chosen breakpoint locations.

\subsection{Distribution of Hyperresidues in Database and Family}

Let $R=\left\langle r_{1}, r_{2}, \cdots, r_{c}\right\rangle$ be a "hyperresidue," a $c$-tuple of amino acid types (e.g. 〈Ala, Val, Ile $\rangle$ ). Intuitively speaking, the more frequently a particular hyperresidue occurs in functional proteins, the more important it is expected to be for their folding and function. We can estimate the overall probability $p$ of hyperresidues from their frequencies in the database $\mathcal{D}$ of protein sequences and corresponding structures:

$$
p(R)=(\# R \text { in } \mathcal{D}) /|\mathcal{D}|,
$$

where $|\mathcal{D}|$ represents the number of tuple instances in the database. When considering a specific protein family $\mathcal{F}$ with a multiple sequence alignment and shared structure, we can estimate position-specific (i.e., for edge $e$ ) probability of a hyperresidue:

$$
p_{e}(R)=(\# R \text { at } e \text { in } \mathcal{F}) /|\mathcal{F}|,
$$

where $|\mathcal{F}|$ is the number of tuple instances at specific positions in the family MSA, i.e. the number of sequences in the family MSA.

Estimation of probabilities from frequencies is valid only if the frequencies are large. Thus the general probability estimated from the whole database (Eq. 1) is more robust than the position-specific from a single family (Eq. 2). However, family-specific information is more valuable as it captures the evolutionarilypreserved interactions in that family. To combine these two aspects, we adopt the treatment of sparse data sets proposed by Sippl [25]:

$$
q_{e}(R)=\omega_{1} \cdot p(R)+\omega_{2} \cdot p_{e}(R),
$$

but employing weights suitable for our problem:

$$
\omega_{1}=1 /(1+|\mathcal{F}| \rho) \text { and } \omega_{2}=1-\omega_{1},
$$

where $\rho$ is a user-specified parameter that determines the relative contributions of database and family. Note that when $\rho=0, q_{e}(R)=p(R)$ and the familyspecific information is ignored; whereas when $\rho=\infty, q_{e}(R)=p_{e}(R)$ and the database information is ignored. Using a suitable value of $\rho$, we will obtain a probability distribution that is close to the overall database distribution for a small family but approximates the family distribution for a large one. 


\subsection{Multi-order Potential Score for Hyperresidues}

Since we have multi-order edges, with lower-order subsets included alongside their higher-order supersets, we must ensure that these edges are not redundant. In other words, a higher-order edge should only include information not captured by its lower-order constituents. The inclusion-exclusion principle ensures nonredundancy in a probability expansion, as Simons et al. 10. demonstrated in the case of protein structure prediction. We define an analogous multi-order potential score for hyperresidues at edges of orders 1, 2, and 3, respectively, as follows:

$$
\begin{aligned}
\phi_{v_{i}}\left(r_{\alpha}\right) & =\log q_{v_{i}}\left(r_{\alpha}\right) \\
\phi_{v_{i} v_{j}}\left(r_{\alpha} r_{\beta}\right) & =\log \frac{q_{v_{i} v_{j}}\left(r_{\alpha} r_{\beta}\right)}{q_{v_{i}}\left(r_{\alpha}\right) \cdot q_{v_{j}}\left(r_{\beta}\right)}, \\
\phi_{v_{i} v_{j} v_{k}}\left(r_{\alpha} r_{\beta} r_{\gamma}\right) & =\log \frac{q_{v_{i} v_{j} v_{k}}\left(r_{\alpha} r_{\beta} r_{\gamma}\right) \cdot q_{v_{i}}\left(r_{\alpha}\right) \cdot q_{v_{j}}\left(r_{\beta}\right) \cdot q_{v_{k}}\left(r_{\gamma}\right)}{q_{v_{i} v_{j}}\left(r_{\alpha} r_{\beta}\right) \cdot q_{v_{i} v_{k}}\left(r_{\alpha} r_{\gamma}\right) \cdot q_{v_{j} v_{k}}\left(r_{\beta} r_{\gamma}\right)} .
\end{aligned}
$$

Here, $\phi_{v_{i}}\left(r_{\alpha}\right)$ captures residue conservation at $v_{i} ; \phi_{v_{i} v_{j}}\left(r_{\alpha} r_{\beta}\right)$ captures pairwise hyperconservation and is zero if $v_{i}$ and $v_{j}$ are not in contact or their residue types are completely independent; $\phi_{v_{i} v_{j} v_{k}}\left(r_{\alpha} r_{\beta} r_{\gamma}\right)$ captures 3-way hyperconservation and is zero if $v_{i}, v_{j}$, and $v_{k}$ are not in contact or their residue types are completely independent. The potential score of higher-order hyperresidues can be defined similarly. The potential score of a higher-order hyperresidue contains no information redundant with that of its lower-order constituents.

\subsection{Edge Weights}

In the hypergraph model, edge weights measure evolutionary optimization of higher-order interactions. For a protein or a set of proteins $\mathcal{S} \subseteq \mathcal{F}$, we can evaluate the significance of an edge as the average potential score of the hyperresidues appearing at the positions forming the edge:

$$
w(e)=\sum_{R} \frac{\# R \text { at } e \text { in } \mathcal{S}}{|\mathcal{S}|} \cdot \phi_{e}(R) .
$$

\subsection{Edge Weights for Recombination}

A particular form of edge weights serves as a guide for breakpoint selection in site-directed recombination. Suppose a set $\mathcal{S} \subseteq \mathcal{F}$ of parents is to be recombined at a set $X=\left\{x_{1}, x_{2}, \cdots, x_{n}\right\}$ of breakpoints, where $x_{t}=v_{i}$ indicates that breakpoint $x_{t}$ is between residues $v_{i}$ and $v_{i+1}$. We can view recombination as a two-step process: decomposing followed by recombining. In the decomposing step, each protein sequence is partitioned into $n+1$ intervals according to the breakpoints, and the hypergraph is partitioned into $n+1$ disjoint subgraphs by removing all edges spanning a breakpoint. The impact of this decomposition can be individually assessed for each edge, using Eq. 8 for the parents $\mathcal{S}$. 
In the recombining step, edges removed in the decomposing step are reconstructed with new sets of hyperresidues according to all combinations of parent fragments. The impact of this reconstruction can also be individually assessed for each edge, yielding a breakpoint-specific weight:

$$
w(e, X)=\sum_{R} \frac{\# R \text { at } e \text { in } \mathcal{L}}{|\mathcal{L}|} \cdot \phi_{e}(R) .
$$

In this case, the potential score of hyperresidue $R$ is weighted by the amount of its representation in the library $\mathcal{L}$. Note that we need not actually enumerate the set of hybrids (which can be combinatorially large) in order to determine the weight, as the frequencies of the residues at the positions are sufficient to compute the frequencies of the hyperresidues.

The combined effect of the two-step recombination process on an individual edge, the edge perturbation, is then defined as the change in edge weight:

$$
\Delta w(e, X)=w(e)-w(e, X) .
$$

If all vertices of $e$ are in one fragment, we have $w(e)=w(e, X)$ and $\Delta w(e, X)=0$. The edge perturbation thus integrates essential information from the database, family, parent sequences, and breakpoint locations.

\section{Optimization of Breakpoint Locations}

Given parent sequences, a set of breakpoints determines a hybrid library. The quality of this hybrid library can be measured by the total perturbation to all edges due to the breakpoints. The hypothesis is that the lower the perturbation, the higher the representation of folded and functional hybrids in the library. We formulate the breakpoint selection problem as follows.

Problem 1. $c$-RECOMB. Given $G_{c}=\left(V, E_{c}, w\right)$ and a positive integer $n$, choose a set of breakpoints $X=\left\{x_{1}, x_{2}, \cdots, x_{n}\right\}$ minimizing $\sum_{e \in E_{c}} \Delta w(e, X)$.

Recall from Sec. 2 that $G_{c}$ represents a hypergraph with edge order uniformly $c$ (where edges with order less than $c$ are also represented as order- $c$ edges).

This hypergraph partitioning problem is significantly more specific than general hypergraph partitioning, so it is interesting to consider its algorithmic difficulty. As as we will see in Sec. 3.1. $c-R E C O M B$ is NP-hard for $c=4$ (and thus also for $c>4$ ), although we provide polynomial-time solutions for $c=2$ in Sec. 3.2 and $c=3$ in Sec. 3.4 .

A special case of $c-R E C O M B$ provides an efficient heuristic approach to minimize the overall perturbation. By minimizing the total weight of all edges $E_{X}$ removed in the decomposing step, fewer interactions need to be recovered in the recombining step.

Problem 2. $c$-DECOMP. Given $G_{c}=\left(V, E_{c}, w\right)$ and a positive integer $n$, choose a set of breakpoints $X=\left\{x_{1}, x_{2}, \cdots, x_{n}\right\}$ minimizing $\sum_{e \in E_{X}} w(e)$.

$c$-DECOMP could also be useful in identifying modular units in protein structures, in which case there is no recombining step. 


\section{$3.1 \quad$ NP-Hardness of $4-R E C O M B$}

4-RECOMB is combinatorial in the set $X$ of breakpoints and the possible configurations they can take relative to each edge. The number of possible libraries could be huge even with a small number of breakpoints (e.g. choosing 7 breakpoints from 262 positions for beta-lactamase results in on the order of $10^{13}$ possible configurations). The choices made for breakpoints are reflected in whether or not there is a breakpoint between each pair of sequentially-ordered vertices of an edge, and thus in the perturbation to the edge. We first give a decision version of 4-RECOMB as follows and then prove that it is NP-hard. Thus the related optimization problem is also NP-hard. Our reduction employs general hypergraphs; analysis in the geometrically-restricted case remains interesting future work.

Problem 3. 4-RECOMB-DEC. Given $G_{4}=\left(V, E_{4}, w\right)$, a positive integer $n$, and an integer $W$, does there exist a set of breakpoints $X=\left\{x_{1}, x_{2}, \cdots, x_{n}\right\}$ such that $\sum_{e \in E_{4}} \Delta w(e, X) \leq W$.

\section{Theorem 3.1. 4-RECOMB-DEC is NP-hard.}

Proof. We reduce from $3 S A T$. Let $\phi=C_{1} \wedge C_{2} \wedge \cdots \wedge C_{k}$ be a boolean formula in 3 -CNF with $k$ clauses. We shall construct a hypergraph $G_{4}=\left(V, E_{4}, w\right)$ such that $\phi$ is satisfiable iff there is a $4-R E C O M B-D E C$ solution for $G_{4}$ with $n=3 k$ breakpoints and $W=-\left|E_{4}\right|$. (See Fig. 2). For clause $C_{i}=\left(l_{i, 1} \vee l_{i, 2} \vee l_{i, 3}\right)$ in $\phi$, add to $V$ four vertices in sequential order $v_{i, 1}, v_{i, 2}, v_{i, 3}$, and $v_{i, 4}$. Elongate $V$ with $3 k$ trivial vertices ( $v_{j}^{\prime}$ in Fig. 2), where we can put trivial breakpoints that cause no perturbation. Let us define predicate $b(i, s, X)=v_{i, s} \in X$ for $s \in\{1,2,3\}$, indicating whether or not there is a breakpoint between $v_{i, s}$ and $v_{i, s+1}$. We also use indicator function $I$ to convert a boolean value to 0 or 1 . We construct $E_{4}$ with three kinds of edges: (1) For the 4-tuple of vertices for clause $C_{i}$, add an edge $e=\left\langle v_{i, 1}, v_{i, 2}, v_{i, 3}, v_{i, 4}\right\rangle$ with $\Delta w(e, X)=-I\{b(i, 1, X) \vee$ $b(i, 2, X) \vee b(i, 3, X)\}$. (2) If two literals $l_{i, s}$ and $l_{j, t}$ are identical, add an edge $e=$ $\left\langle v_{i, s}, v_{i, s+1}, v_{j, t}, v_{j, t+1}\right\rangle$ with $\Delta w(e, X)=-I\{b(i, s, X)=b(j, t, X)\}$. (3) If two literals $l_{i, s}$ and $l_{j, t}$ are complementary, add an edge $e=\left\langle v_{i, s}, v_{i, s+1}, v_{j, t}, v_{j, t+1}\right\rangle$ with $\Delta w(e, X)=-I\{b(i, s, X) \neq b(j, t, X)\}$.

There are $7 k$ vertices and at most $k+3\left(\begin{array}{c}k \\ 2\end{array}\right)=O\left(k^{2}\right)$ edges, so the construction takes polynomial time. It is also a reduction. First, if $\phi$ has a satisfying assignment, choose breakpoints $X=\left\{v_{i, s} \mid l_{i, s}\right.$ is TRUE $\}$ plus additional breakpoints between the trivial vertices to reach $3 k$ total. Since each clause is satisfied, one of its literals is true, so there is a breakpoint in the corresponding edge $e$ and its perturbation is -1 . Since literals must be used consistently, type 2 and 3 edges also have -1 perturbation. Thus $4-R E C O M B-D E C$ is satisfied with $n=3 k$ and $W=-\left|E_{4}\right|$. Conversely, if there is a $4-R E C O M B-D E C$ solution with breakpoints $X$, then assign truth values to variables such that $l_{i, s}=b(i, s, X)$ for $s \in\{1,2,3\}$ and $i \in\{1,2, \cdots, k\}$. Since perturbation to type 1 edges is -1 , there must be at least one breakpoint in each clause vertex tuple, and thus a true literal in the clause. Since perturbation to type 2 and 3 edges is -1 , literals are used consistently. 


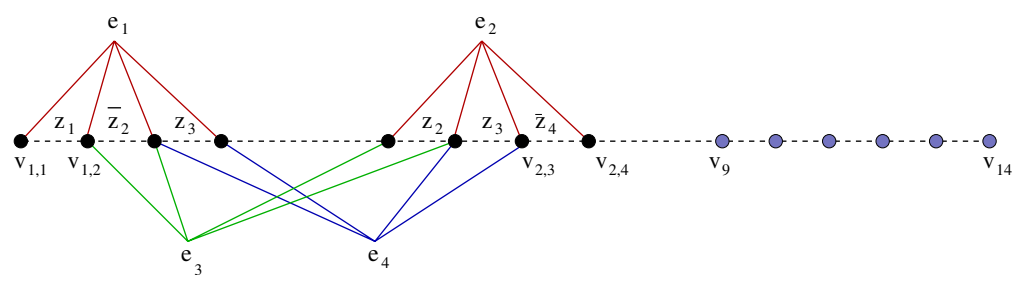

Fig. 2. Construction of hypergraph $G_{4}=\left(V, E_{4}, w\right)$ from an instance of $3 S A T \phi=$ $\left(z_{1} \vee \bar{z}_{2} \vee z_{3}\right) \wedge\left(z_{2} \vee z_{3} \vee \bar{z}_{4}\right)$. Type 1 edges $e_{1}$ and $e_{2}$ ensure the satisfaction of clauses $(-1$ perturbation iff there is a breakpoint iff the literal is true and the clause is satisfied), while type 3 edge $e_{3}$ and type 2 edge $e_{4}$ ensure the consistent use of literals $(-1$ perturbation iff the breakpoints are identical or complementary iff the variable has a single value).

We note that 4-RECOMB-DEC is in NP, since given a set of breakpoints $X$ for parents $\mathcal{S}$ we can compute $\Delta w(e, X)$ for all edges in polynomial time $\left(O\left(\mathcal{S}^{4} E\right)\right)$, and then must simply sum and compare to a provided threshold.

\subsection{Dynamic Programming Framework}

Despite the NP-hardness of the general sequentially-constrained hypergraph partitioning problem $c-R E C O M B$, the structure of the problem (i.e. the sequential constraint) leads to efficient solutions for some important cases. Suppose we are adding breakpoints one by one from left to right ( $\mathrm{N}$ - to $\mathrm{C}$-terminal) in the sequence. Then the additional perturbation to an edge $e$ caused by adding breakpoint $x_{t}$ given previous breakpoints $X_{t-1}=\left\{x_{1}, x_{2}, \cdots, x_{t-1}\right\}$ can be written:

$$
\Delta \Delta w\left(e, X_{t-1}, x_{t}\right)=\Delta w\left(e, X_{t}\right)-\Delta w\left(e, X_{t-1}\right)
$$

where $X_{0}=\emptyset$ and the additional perturbation caused by the first breakpoint is $\Delta \Delta w\left(e, X_{0}, x_{1}\right)=\Delta w\left(e, X_{1}\right)$. Reusing notation, we indicate the total additional perturbation to all edges as $\Delta \Delta w\left(E, X_{t-1}, x_{t}\right)$. Now, if the value of $\Delta \Delta w\left(E, X_{t-1}, x_{t}\right)$ can be determined by the positions of $x_{t-1}$ and $x_{t}$, independent of previous breakpoints, then we can adopt the dynamic programming approach shown below. When the additional perturbation depends only on $x_{t-1}$ and $x_{t}$, we write it as $\Delta \Delta w\left(E, x_{t-1}, x_{t}\right)$ to indicate the restricted dependence.

Let $d[t, \tau]$ be the minimum perturbation caused by $t$ breakpoints with the rightmost at position $\tau$. If, for simplicity, we regard the right end of the sequence as a trivial breakpoint that causes no perturbation, then $d[n+1,|V|]$ is the minimum perturbation caused by $n$ breakpoints plus this trivial one, i.e. the objective function for Problem [1. We can compute $d$ recursively:

$$
d[t, \tau]= \begin{cases}\Delta w(E,\{\tau\}), & \text { if } t=1 \\ \min _{\lambda \leq \tau-\delta}\{d[t-1, \lambda]+\Delta \Delta w(E, \lambda, \tau)\}, & \text { if } t \geq 2 .\end{cases}
$$

where $\delta$ is a user-specified minimum sequential distance between breakpoints. The recurrence can be efficiently computed bottom-up in a dynamic programming style, due to its optimal substructure. In the following, we instantiate this 


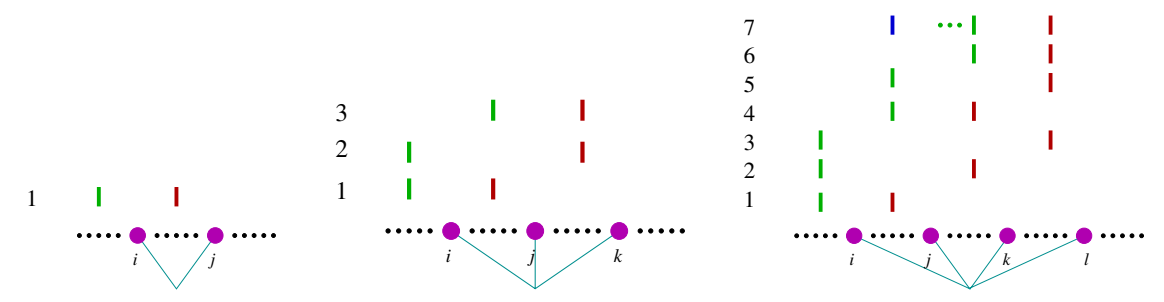

Fig. 3. All breakpoint configurations that cause additional perturbation to an edge as breakpoints in a $c-R E C O M B$ problem are added one by one from left to right in the sequence. The dynamic programming formulation requires that we be able to distinguish the configurations from each other and from configurations with no additional perturbation. For an order- 2 edge $\left\langle v_{i}, v_{j}\right\rangle$, there is additional perturbation if and only if the current breakpoint (red) is added between $v_{i}$ and $v_{j}$ and the previous breakpoint (green) is to the left of $v_{i}$. Similarly, the configurations on an order-3 edge $\left\langle v_{i}, v_{j}, v_{k}\right\rangle$ can be distinguished by the positions of the current breakpoint (red) and the preceding one (green) with respect to the intervals $\left[v_{i}, v_{j}\right]$ and $\left[v_{j}, v_{k}\right]$. However, for an order-4 edge, configurations 6 and 7 are ambiguous with respect to the intervals of $\left\langle v_{i}, v_{j}, v_{k}, v_{l}\right\rangle$. We cannot be certain about the existence of a potential breakpoint between $v_{i}$ and $v_{j}$ (blue) without potentially looking back at all previous breakpoints (green ellipses).

dynamic programming formulation with different forms of $\Delta \Delta w$ for different cases of $c-R E C O M B$ and $c-D E C O M P$. Due to space limitations, time complexity analyses are omitted.

The special case of 2-DECOMP (disruption of pairwise interactions) has been previously solved as a shortest path problem 24. A complexity analysis accounting for both the edge weight calculation and dynamic programming shows that the total time is $O\left(\mathcal{S}^{2} E+V E+n V^{2}\right)$.

The instantiation for $2-R E C O M B$ is as follows. Each order-2 edge $\left\langle v_{i}, v_{j}\right\rangle$ has two states: either there is breakpoint between $v_{i}$ and $v_{j}$ or not (Fig. 33). The state of $e$ is changed by adding breakpoint $x_{t}$ iff $x_{t-1}<v_{i}<x_{t}<v_{j}$. Thus the additional perturbation caused by adding $x_{t}$ can be determined by the positions of $x_{t-1}$ and $x_{t}$, and is independent of previous breakpoints. Our dynamic programming framework Eq. 12 is therefore applicable to 2-RECOMB; the time complexity is $O\left(\mathcal{S}^{2} E+V E+n V^{2}\right)$.

\subsection{Reduction from $c-D E C O M P$ to $2-D E C O M P$}

A significant property of our multi-order potential score (Sec. 2.2) is that the score of a higher-order edge captures only higher-order hyperconservation and contains no information about its lower-order constituents. Thus in the decomposition phase, a higher-order edge is broken if there is a breakpoint anywhere in the set of residue positions it spans. The lack of breakpoints between any adjacent pair of its vertices will be captured by the weight of the appropriate lower-order constituent edge. By this reasoning, we can reduce the $c-D E C O M P$ problem to the 2-DECOMP problem: given hypergraph $G_{c}=\left(V_{c}, E_{c}, w_{c}\right)$, construct graph $G_{2}=\left(V_{2}, E_{2}, w_{2}\right)$ such that $V_{2}=V_{c}$ and each edge $e_{c}=\left\langle v_{1}, v_{2}, \cdots, v_{c}\right\rangle \in E_{c}$ is 
mapped to an edge $e_{2}=\left\langle v_{1}, v_{c}\right\rangle \in E_{2}$ connecting the first and last vertex of $e_{c}$, putting weight $w_{c}\left(e_{c}\right)$ on $w_{2}\left(e_{2}\right)$. There is a breakpoint decomposing $e_{c}$ in $G_{c}$ iff there is one decomposing $e_{2}$ in $G_{2} . G_{2}$ can be constructed in $O(V+E)$ time, and optimal solutions for $c$-DECOMP on $G_{c}$ correspond to optimal solutions for 2$D E C O M P$ on $G_{2}$. Under this reduction (which adds only $O(E)$ computation), the total time complexity for $c-D E C O M P$ is $O\left(\mathcal{S} E+V E+n V^{2}\right)$. Thus protein modules can be computed under $c-D E C O M P$ in polynomial time for any order of edge.

\subsection{Dynamic Programming for 3-RECOMB}

We have seen that the $c-R E C O M B$ problem is NP-hard when $c \geq 4$ (Sec. 3.1) and solvable in polynomial time when $c=2$ (Sec. 3.2). In this section, we instantiate our dynamic programming framework to give a polynomial-time solution when $c=3$.

An order-3 edge has four possible states, according to whether or not there is at least one breakpoint between each pair of its vertices listed in sequential order. As Fig. 3 illustrates, given only $x_{t-1}$ and $x_{t}$, all breakpoint configurations that cause additional perturbation can be uniquely determined, and the additional perturbation can be computed as in Eq. 11. This edge perturbation calculation meets the restriction required for our dynamic programming framework, and Eq. 12 and be used to optimize 3-RECOMB in $O\left(\mathcal{S}^{3} E+V E+n V^{2}\right)$ time.

\subsection{Stochastic Dynamic Programming for $4-R E C O M B$}

Tetrahedra are natural building blocks of 3D structures, and Delaunay tetrahedra in the protein core have been shown to capture interactions important for protein folding [8. Our results below show significant information in general order-4 hyperconservation. In order to solve $4-R E C O M B$ problems, we develop here a heuristic approach based on stochastic dynamic programming. Unlike 2RECOMB and 3-RECOMB, the additional perturbation of a breakpoint cannot always be determined by reference just to the current and previous breakpoint locations. As Fig. 3 shows, given $x_{t-1}$ and $x_{t}$, there is ambiguity only between configurations 6 and 7.

We can still employ the dynamic programming framework if we move from a deterministic version, in which both the additional perturbation and next state are known, to a stochastic version, in which they are predicted as expected values. In the ambiguous case of configurations 6 and 7 with $t \geq 2$, let us assume that breakpoints before $x_{t-1}$ are uniformly distributed in the sequence. Then the probability of finding no breakpoint between $v_{i}$ and $v_{j}$, i.e. being in configuration 6 rather than 7 , is

$$
p=\left(1-\frac{v_{j}-v_{i}}{x_{t-1}}\right)^{t-2},
$$

since $\frac{v_{j}-v_{i}}{x_{t-1}}$ is the probability of a breakpoint being located between $v_{i}$ and $v_{j}$ and $t-2$ is the number of breakpoints before position $x_{t-1}$. Thus for the ambiguous cases, the expected additional perturbation to $e$ caused by adding $x_{t}$ is

$$
\Delta \Delta w\left(e, x_{t-1}, x_{t}, t\right)=p \cdot \Delta \Delta w_{6}\left(e, x_{t-1}, x_{t}\right)+(1-p) \cdot \Delta \Delta w_{7}\left(e, x_{t-1}, x_{t}\right),
$$


where the subscript indicates the configuration. Note that, unlike our previous formulations, the additional perturbation depends on the number of previous breakpoints. Thus the time complexity of this stochastic dynamic programming is increased to $O\left(\mathcal{S}^{4} E+n V E+n V^{2}\right)$. This stochastic dynamic programming technique can also be applied to $c>4 c-R E C O M B$ problems, but the effectiveness of the approximation is expected to decrease with an increasing number of ambiguous states.

\section{Results and Discussion}

We demonstrate our hypergraph model and recombination planning algorithms in analysis of the beta-lactamase protein family, since previous site-directed recombination experiments have employed beta-lactamase parents TEM-1 and PSE-4 23. We identified 123 beta-lactamases for $\mathcal{F}$, including TEM-1 and PSE-4, with no more than $80 \%$ sequence identity, and constructed a multiple sequence alignment with at most $20 \%$ gaps in any sequence. PDB file 1BTL was used as the representative family structure. Vertices were considered as located at the average position of non-hydrogen side-chain atoms, and edges formed for sets of vertices whose positions were within $8 \AA$ of each other.

For the database $\mathcal{D}$, we started with a subset of sequences culled from the protein data bank according to structure quality (R-factor less than 0.25 ) and mutual sequence identity (at most $60 \%$ ) by PISCES [26]. To minimize the effect of structural errors on statistical results, chains with nonconsecutive residue numbers, gaps ( $\mathrm{C}^{\alpha}-\mathrm{C}^{\alpha}$ distance greater than $4.2 \AA$ between consecutive residues), or incorrect atom composition of residues were excluded [9]. This left 687 chains. Contact maps were constructed as with the family.

We first considered the information content in higher-order interactions. Fig. 4 shows the distributions of hyperresidue potential scores in both the database and family, for increasing hyperresidue order. By the non-redundant decomposition, a higher-order potential score would be 0 if the lower-order terms were independent. Non-zero $\phi(R)$ scores represent positive and negative correlation. The figure shows that there is clearly information in the sets of higher-order terms. Note that the family distributions are biased ( $\mu$ not at zero), presumably because many sets of amino acid types are not observed in the MSA. Family distributions are also more informative than database ones (larger $\sigma$ for all orders). Dicysteine pairs are expected to be particularly informative (i.e. cysteines in disulfides are not independent), as reflected in the clear outliers marked in the $c=2$ database histogram; there are no disulfides in the beta-lactamase family.

A limited amount of data is currently available for evaluating the experimental effectiveness of a recombination plan. Here, we use the beta-lactamase hybrid library of [23. For each hybrid in the library, we computed both the total potential score and the mutation level. The total potential score is the sum, over all edges up to order-4, of the edge potential (Eq. [5- 7) for the residues in the hybrid sequence. The mutation level is the number of residues in the hybrid that differ from the closest parent. While hybrids with small mutation levels are expected 

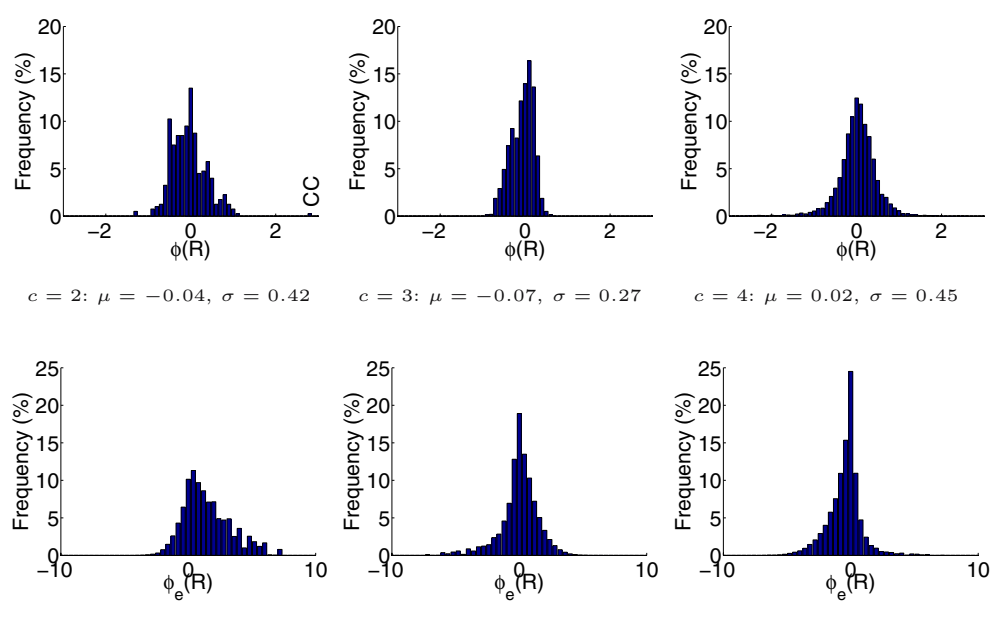

$c=2: \mu=1.58, \sigma=1.98$

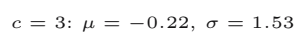

$c=4: \mu=-0.20, \sigma=1.34$

Fig. 4. Multi-order potential scores, derived from the database (top) and the betalactamase family (bottom). For each order $c$ of hyperresidues, the distribution of potential scores is shown (pooled over all edges for the family version).

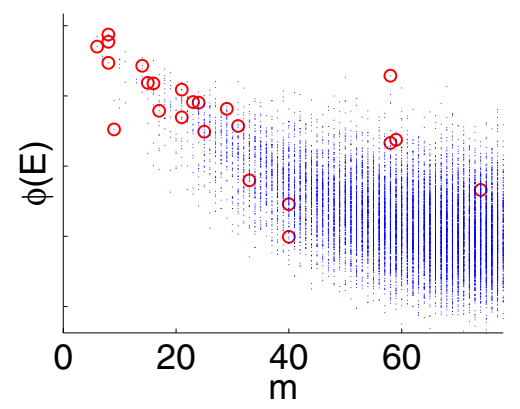

Fig. 5. Potential score $\phi(E)$ (sum over all interactions up to order-4) vs. mutation level $m$ (\# residues different from the closest parent) for all hybrids in a beta-lactamase library. Blue dots indicate hybrids, and red circles those determined to be functional [23].

to be functional, our potential yields high scores for the functional hybrids at high mutation levels (Fig. [5).

Next we applied our dynamic programming algorithms to optimize 7 -break point sets for different beta-lactamase parents (Fig. 6), using minimum effective fragment length $\delta=10$, database/family weight $\rho=0.01$, and maximum order of edges $c=3$. We found the results to be insensitive to $\rho$, beyond very small values placing all the emphasis on the database (data not shown). In the 1-parent case, the plan amounts to decomposing the protein (PDB file 1BTL as representative family structure) into modules preserving multi-order interactions. The 2-parent and 12-parent cases illustrated here would be useful in site-directed recombination experiments. We note that some locations can "float" due to parent 

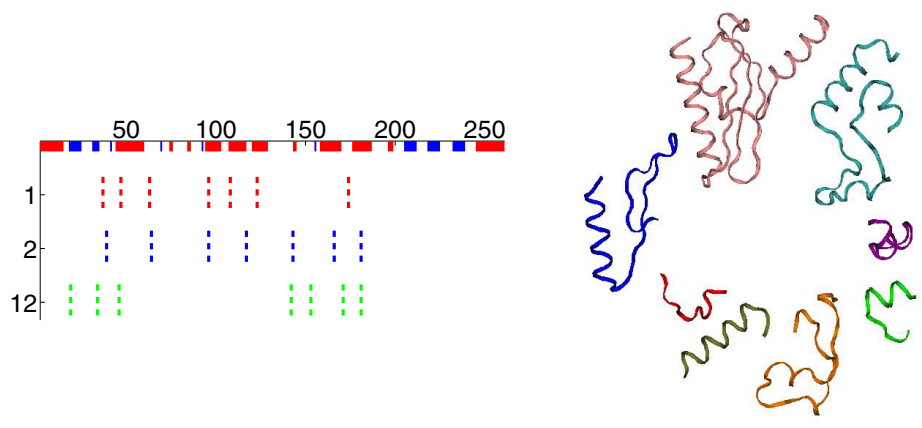

Fig. 6. Beta-lactamase breakpoint optimization. (Left) Optimized breakpoint locations when planning with 1,2 , or 12 parents. The sequence is labeled with residue index, with helices in red and $\beta$-sheets in blue. (Right) 3D structure fragments (PDB id: 1BTL) according to optimized breakpoint locations for the 1-parent case.

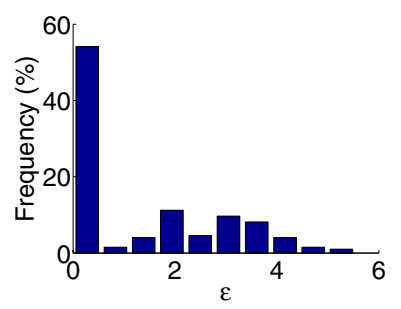

Fig. 7. Distribution of differences in edge perturbations between the two ambiguous configurations (6 and 7) in 4-RECOMB for the beta-lactamases TEM-1 and PSE-4. Differences $(\varepsilon)$ are expressed in standard deviations units.

sequence identity (e.g. in positions $17-20$ with 2 parents). These all represent viable experiment plans, optimizing multi-order interactions according to sequence characteristics of different parents.

Finally, we considered the error that could be caused by the stochastic approximation in solving 4-RECOMB. Fig. 7 shows the distribution, over all order- 4 edges, of differences in perturbations between the ambiguous states. The differences are expressed in terms of perturbation standard deviations $\varepsilon=\frac{\left|\Delta \Delta w_{6}-\Delta \Delta w_{7}\right|}{\left(\operatorname{std}\left(\Delta \Delta \mathrm{w}_{6}\right)+\operatorname{std}\left(\Delta \Delta \mathrm{w}_{7}\right)\right) / 2}$. Edges with identical residues at $v_{i}$ or $v_{j}$ are excluded, since the perturbation is necessarily the same. Even so, in a majority of cases the heuristic would lead to no or very small error. Thus the stochastic dynamic programming will provide a near optimal solution, which makes it reasonable to include 4 -way interactions in practice.

\section{Conclusion}

We have developed a general hypergraph model of multi-order residue interactions in proteins, along with algorithms that optimize site-directed recombination experiments under the model. The model has a number of other potential 
applications for which multi-order interactions are significant, after suitable parameterization, including prediction of $\Delta \mathrm{G}^{\circ}$ of unfolding, $\Delta \Delta \mathrm{G}^{\circ}$ of mutagenesis, and modularity of protein structures. The algorithms likewise can be employed using potentials that incorporate additional information (e.g. weighted for active sites). Interesting future work includes selection of parent sequences, separation of stability-critical and functionality-critical multi-residue interactions, interpretation of experimental data, and feedback of experimental results to subsequent rounds of planning.

\section{Acknowledgments}

We thank Dr. Bruce Craig (Statistics, Purdue), Shobha Potluri and John Thomas (CS, Dartmouth), and Michal Gajda (IIMCB, Poland) for stimulating discussion. This work was supported in part by an NSF CAREER award to CBK (IIS-0444544) and an NSF SEIII grant to CBK, AMF, and Bruce Craig (IIS-0502801).

\section{References}

1. Tanaka, S., Scheraga, H.: Medium and long range interaction parameters between amino acids for predicting three dimensional strutures of proteins. Macromolecules 9 (1976) 945-950

2. Miyazawa, S., Jernigan, R.: Estimation of effective interresidue contact energies from protein crystal structures: Quasi-chemical approximation. Macromolecules 18 (1985) 531-552

3. Maiorov, V., Crippen, G.: Contact potential that recognizes the correct folding of globular proteins. J. Mol. Biol. 227 (1992) 876-88

4. Simons, K.T., Kooperberg, C., Huang, E., Baker, D.: Assembly of protein tertiary structures from fragments with similar local sequences using simulated annealing and Bayesian scoring functions. J. Mol. Biol. 268 (1997) 209-225

5. Kihara, D., Lu, H., Kolinski, A., Skolnick, J.: TOUCHSTONE: an ab initio protein structure prediction method that uses threading-based tertiary restraints. PNAS 98 (2001) 10125-10130

6. Godzik, A.: Fold recognition methods. Methods Biochem. Anal. 44 (2003) 525-546

7. Betancourt, M., Thirumalai, D.: Pair potentials for protein folding: Choice of reference states and sensitivity of predictive native states to variations in the interaction schemes. Protein Sci. 8 (1999) 361-369

8. Carter Jr., C., LeFebvre, B., Cammer, S., Tropsha, A., Edgell, M.: Four-body potentials reveal protein-specific correlations to stability changes caused by hydrophobic core mutations. J. Mol. Biol. 311 (2001) 621-638

9. Krishnamoorthy, B., Tropsha, A.: Development of a four-body statistical pseudopotential to discriminate native from non-native protein conformations. Bioinformatics 19 (2003) 1540-1548

10. Simons, K., Ruczinski, I., Kooperberg, C., Fox, B., Bystroff, C., Baker, D.: Improved recognition of native-like protein structures using a combination of sequence-dependent and sequence-independent features of proteins. PROTEINS: Structure, Function, and Genetics 34 (1999) 82-95 
11. Gobel, U., Sander, C., Schneider, R., Valencia, A.: Correlated mutations and residue contacts in proteins. PROTEINS: Structure, Function, and Genetics 18 (1994) 309-317

12. Lockless, S., Ranganathan, R.: Evolutionarily conserved pathways of energetic connectivity in protein families. Science 286 (1999) 295-299

13. Thomas, J., Ramakrishnan, N., Bailey-Kellogg, C.: Graphical models of residue coupling in protein families. In: 5th ACM SIGKDD Workshop on Data Mining in Bioinformatics (BIOKDD). (2005)

14. Stemmer, W.: Rapid evolution of a protein in vitro by DNA shuffling. Nature $\mathbf{3 7 0}$ (1994) 389-391

15. Ostermeier, M., Shim, J., Benkovic, S.: A combinatorial approach to hybrid enzymes independent of DNA homology. Nat. Biotechnol. 17 (1999) 1205-9

16. Lutz, S., Ostermeier, M., Moore, G., Maranas, C., Benkovic, S.: Creating multiplecrossover DNA libraries independent of sequence identity. PNAS 98 (2001) $11248-53$

17. Sieber, V., Martinez, C., Arnold, F.: Libraries of hybrid proteins from distantly related sequences. Nat. Biotechnol. 19 (2001) 456-60

18. Voigt, C., Martinez, C., Wang, Z., Mayo, S., Arnold, F.: Protein building blocks preserved by recombination. Nat. Struct. Biol. 9 (2002) 553-558

19. O'Maille, P., Bakhtina, M., Tsai, M.: Structure-based combinatorial protein engineering (SCOPE). J. Mol. Biol. 321 (2002) 677-691

20. Aguinaldo, A., Arnold, F.: Staggered extension process (StEP) in vitro recombination. Methods Mol. Biol. 231 (2003) 105-110

21. Coco, W.: RACHITT: Gene family shuffling by random chimeragenesis on transient templates. Methods Mol. Biol. 231 (2003) 111-127

22. Castle, L., Siehl, D., Gorton, R., Patten, P., Chen, Y., Bertain, S., Cho, H.J., Duck, N., Wong, J., Liu, D., Lassner, M.: Discovery and directed evolution of a glyphosate tolerance gene. Science 304 (2004) 1151-4

23. Meyer, M., Silberg, J., Voigt, C., Endelman, J., Mayo, S., Wang, Z., Arnold, F.: Library analysis of SCHEMA-guided protein recombination. Protein Sci. 12 (2003) 1686-93

24. Endelman, J., Silberg, J., Wang, Z.G., Arnold, F.: Site-directed protein recombination as a shortest-path problem. Protein Eng., Design and Sel. 17 (2004) 589-594

25. Sippl, M.: Calculation of conformational ensembles from potentials of mean force. an approach to the knowledge-based prediction of local structures in globular proteins. J. Mol. Biol. 213 (1990) 859-883

26. Wang, G., R. L. Dunbrack, J.: Pisces: a protein sequence culling server. Bioinformatics 19 (2003) 1589-1591 